\title{
USOS E SIGNIFICADOS DA QUALIDADE DE VIDA NOS DISCURSOS CONTEMPORÂNEOS DE SAÚDE
}

\author{
USES AND MEANINGS OF QUALITY OF LIFE IN THE CONTEMPORARY DISCOURSE ON HEALTH
}

\author{
Gabriel de Freitas Gimenes ${ }^{1}$
}

Resumo $O$ presente artigo se propõe a fazer uma análise do uso generalizado da expressão 'qualidade de vida' nas linguagens técnicas e cotidianas nas sociedades ocidentais contemporâneas. A ampla utilização desse termo é acompanhada por uma indeterminação de seu significado e por uma concepção naturalizada de sua origem. Objetivo, então, analisar os processos pelos quais o termo qualidade de vida foi impregnado de sentido na nossa cultura, assim como argumentar que a grande relevância simbólica adquirida contemporaneamente pela qualidade de vida nas ciências biomédicas, nas políticas de saúde e nas práticas individuais relativas à saúde está relacionada com um mais amplo processo de inserção dos aspectos biológicos da vida no campo político. Dessa forma, apresento brevemente o surgimento e o desenvolvimento da biopolítica na modernidade, assim como analiso algumas das características da biopolítica contemporânea, a partir do que são apresentadas as concepções de estilo de vida, promoção de saúde, risco, autonomia e responsabilidade, que, articuladas, constituem o atual campo semântico no qual a saúde é problematizada. Dessa forma, procuro situar a atual preocupação pela qualidade de vida, assim como apresentar alguns dos problemas que se articulam a ela, como a responsabilidade individual pela própria saúde e a medida da qualidade de vida.

Palavras-chave qualidade de vida; saúde; biopolítica.
Abstract This article is an attempt to analyze the widespread use of the term 'quality of life' in everyday language and techniques in contemporary Western societies. The broad use of this term is accompanied by an uncertainty with regard to its meaning and a naturalized conception of its origin. The goal, therefore, is to analyze the processes by which the term quality of life was imbued with meaning in our culture and to argue that the major symbolic meaning acquired contemporaneously by quality of life in the biomedical sciences, health policies, and practices relating to individual health is related to a broader process of integrating biological aspects of life in the political field. Thus, I briefly introduce the emergence and development of biopolitics in modernity, and analyze some of the characteristics of contemporary biopolitics based on how the concepts of lifestyle, promotion of health, risk, autonomy and responsibility are presented which, together, constitute the current semantic field in which health is problematized. Therefore, I seek to situate the current concern for quality of life, as well as to present some of the issues that are linked to it, such as individual responsibility for one's own health and the measure of quality of life.

Keywords quality of life; health; biopolitics. 


\title{
Introdução
}

\author{
Nós nos tornamos os tipos de pessoas que pensam \\ em nosso presente e em nosso futuro em termos da \\ qualidade de nossas vidas biológicas individuais. ${ }^{2}$ \\ (Rose, 2001, p. 22, minha tradução).
}

Qualidade de vida é uma expressão largamente utilizada nos dias de hoje. É muito comum ouvir pessoas comentando sobre mudanças em seus estilos de vida na busca por um aumento de sua qualidade de vida. Proliferam as propagandas dos mais diversos produtos que tentam seduzir seus consumidores com a promessa de aprimoramento da qualidade de vida. Diversas profissões relacionadas à saúde se especializam, atualmente, na busca pela qualidade de vida de seus clientes. No campo mais específico das ciências biomédicas, Belasco e Sesso (2006) identificaram um grande aumento no número de pesquisas relacionadas à qualidade de vida:

Enquanto, em 1973, uma busca utilizando o termo qualidade de vida na MEDLINE encontrava somente cinco artigos, em 1998 esse número passou a 16.256 citações (Sem, Gupchup e Thomas, 1965) e, em 2003, chegou a 53.588 (National Library of Medicine, 2003). A pesquisa indexada no Brasil sobre o assunto cresceu 106\% entre 1981 e 1991. Entre 1990 e 2002, o número de teses sobre QV foi de 9.635 (Instituto Brasileiro de Informação em Ciência e Tecnologia) (Belasco e Sesso, 2006, p. 6-7).

Pode-se observar que a qualidade de vida conquistou contemporaneamente uma hegemonia discursiva tanto na linguagem técnica quanto na linguagem cotidiana. No entanto, como afirmam Minayo, Hartz e Buss, "o termo qualidade de vida aparece sempre com sentido bastante genérico. (...) Isso quer dizer que se a ideia geral de qualidade de vida está presente, precisa ser mais bem explicitada e clarificada" (Minayo, Hartz e Buss, 2000, p. 8).

Apesar ou justamente em decorrência de sua ampla dispersão discursiva, o termo qualidade de vida apresenta grande indeterminação em seu significado. Aparentemente, essa própria indeterminação beneficia sua utilização difusa, uma vez que, como argumenta Matta:

Quanto maior a sua indefinição, maior o seu poder retórico. Ao reunir tantos sentidos, a noção de qualidade de vida perde a capacidade de identificar nexos causais ou interpretativos, servindo para os mais diversos fins (2005, p. 108, grifos meus). 
Dentro dessa indefinição, podem-se esboçar alguns dos fins aos quais a utilização da expressão qualidade de vida atende: técnicos, como conceito articulador de outros conceitos ou de práticas nas ciências biomédicas; econômicos, como aglutinador de valor ou justificador da produção ou compra de produtos e serviços; clínicos, como indicador da eficácia de um procedimento de intervenção ou como medida organizadora de grupos de pacientes; político-administrativos, como medida de avaliação e de comparação de políticas públicas de saúde; e subjetivos, como maneira de perceber e avaliar a própria saúde ou como forma de organizar as possibilidades de seu aprimoramento.

Tal esforço classificatório, entretanto, é apenas um esboço analítico que visa realizar uma primeira localização dos campos e dos usos discursivos da expressão qualidade de vida, uma vez que, em sua utilização difusa e indeterminada, esses diversos usos e campos surgem aglutinados e correlacionados em rede.

Esse potencial articulador e discursivo que a expressão qualidade de vida adquiriu contemporaneamente não surgiu do nada, mas antes foi composto mediante complexos processos, ocorridos simultaneamente em distintos níveis, por meio dos quais ela foi imbuída de sentidos e se generalizou. Entretanto, esses processos são comumente esquecidos ou silenciados, de maneira que a qualidade de vida é apresentada de uma forma naturalizada, como se ela sempre tivesse sido uma preocupação da humanidade, como é possível observar em alguns textos:

A busca pelo significado do termo qualidade de vida $(\mathrm{QV})$ parece ser tão antiga quanto a civilização. Diferentes referenciais filosóficos, desde a Antiguidade, conceituam o que seja vida com qualidade (Belasco e Sesso, 2006, p. 1).

O termo qualidade de vida (QV) é o resultado final de um processo histórico cujas primeiras tentativas conceituais surgem a partir de 384 a.C. (Blay e Merlin, 2006, p. 19).

Essa apresentação da qualidade de vida de forma naturalizada é problemática, principalmente em três aspectos: desgasta e esvazia o termo, uma vez que o descola das contingências históricas e culturais nas quais surgiu e adquiriu significado; corresponde a uma universalização de uma questão que é eminentemente nossa, das sociedades ocidentais contemporâneas; e silencia os processos pelos quais foi tornada possível a construção e a generalização do termo.

As formulações deste artigo procuram distanciar-se dessa concepção naturalizada da qualidade de vida, uma vez que partilham da premissa de 
Matta (2005) de que o surgimento desse termo é o resultado de um "movimento/campo discursivo e político" no qual é possível identificar "uma convergência de interesses, investimentos e disputa" (Matta, 2005, p. 115).

Dessa forma, parto da problematização da qualidade de vida sob três aspectos: o uso generalizado da expressão; a indeterminação do seu significado; e a naturalização de sua concepção. Com base nessas questões, procuro analisar os diversos usos e significados da qualidade de vida, não para organizar o caos existente em sua indeterminação semântica, mas para situá-la nos discursos e práticas contemporâneos que constituem nossa cultura, a fim de tentar compreender como o termo adquiriu tantos significados, e o que ele diz a respeito de nós e de nossas compreensões de saúde e de vida saudável.

Um dos meus principais argumentos é que a constituição desses diversos usos da expressão qualidade de vida em distintos campos discursivos não foi um processo inocente ou linear, mas que, antes, situa-se numa complexa correlação de forças que tornou possível a composição desse problema. A fim de tentar localizar provisoriamente alguns desses campos de saberes/poderes, podem-se situar os seguintes: epistemológico, que procuro discutir pelo movimento de alteração ocorrida na concepção de saúde pelas ciências biomédicas, ao qual denomino deslocamento qualitativo; politico, a partir das mudanças ocorridas nas estratégias biopolíticas contemporâneas, do surgimento da nova saúde pública, do enfraquecimento do papel do Estado de garantir o direito à saúde e do desenvolvimento de medidas globais de avaliação da saúde em consonância com os movimentos hegemônicos de globalização; cultural, que situo nas concepções de risco, estilo de vida e promoção de saúde que se articulam com a emergência, cada vez mais hegemônica, de uma concepção de pessoa enquanto indivíduo autônomo responsável pela gestão de si; e econômico, pela criação de novos nichos de mercado vinculados ao consumo de novos produtos que adquirem valor por meio de suas qualificações como saudáveis ou relativos a estilos de vida saudáveis. A constituição do problema da qualidade de vida pode ser localizada nesses distintos movimentos ocorridos simultaneamente em vários campos de práticas.

Com base no conceito de biopolítica (Foucault, 2008), procuro compreender como ocorreu, na modernidade, a problematização política dos aspectos biológicos da vida, os quais se tornaram questões cruciais da governamentalidade e serviram de fundamento para o desenvolvimento das noções de população, da saúde enquanto direito dos cidadãos e das práticas médicas enquanto estratégias de governo. A partir dessa compreensão, procuro situar brevemente o desenvolvimento das políticas de saúde da modernidade e as características daquelas insurgentes práticas médicas. Em seguida, apresento um breve quadro das principais mudanças ocorridas nas estratégias biopolíticas contemporâneas. Procuro argumentar como ocorreu o deslocamento da questão da saúde de uma esfera mais quantitativa e de massa para outra 
mais qualitativa e individual, na qual podem ser localizadas noções como risco, estilo de vida e promoção de saúde, as quais, articuladas, compõem o campo discursivo em que é tornado possível o problema da qualidade de vida e sua discussão, assim como uma preocupação com ela. Como procuro demonstrar, tal deslocamento não constituiu uma substituição total das questões e preocupações anteriores, antes formou uma nova rede que tanto rearticulou concepções anteriores quanto introduziu novas.

A preocupação com a qualidade de vida individual se aproxima de uma concepção de pessoa muito específica da nossa cultura: a do indivíduo autônomo. Faz-se, então, necessário relacionar os discursos hegemônicos de qualidade de vida com essa concepção de autonomia a partir da qual são apresentadas algumas das práticas relacionadas com esses discursos, como a autoadministração dos riscos e a responsabilidade individual pela própria saúde (Osborne, 2005; Petersen, 2005). Argumento, com base em Rose (2001) e Spink (2007), que a noção de responsabilidade veiculada por esses discursos e práticas assume contornos tanto econômicos quanto morais.

Por fim, realizo uma breve análise dos instrumentos de medida de qualidade de vida. Tais instrumentos, segundo Matta (2005), ao focalizarem a percepção subjetiva do próprio estado de saúde, fornecem dados para a análise da eficácia de práticas de intervenção biomédica, assim como para a análise de políticas de saúde e para o desenvolvimento de estratégias globais de promoção de saúde. Procuro, então, compreender como se tornou possível a utilização desses instrumentos e como eles rearticulam noções tais como objetividade e subjetividade.

Uma vez que a qualidade de vida é uma expressão em constante negociação e disputa, cabe salientar que, ao comentar sobre a moralização das práticas de saúde individuais, a totalização da saúde enquanto bem e a consequente culpabilização dos não saudáveis, refiro-me apenas aos discursos e práticas hegemônicos de qualidade de vida. Compreendo esses como aqueles largamente veiculados pela grande mídia e pelas propagandas dos produtos do bem-estar, ao passo que o leque dos possíveis discursos e práticas contra-hegemônicos que também podem se relacionar com a qualidade de vida foram deixados para uma análise posterior.

\section{Biopolítica e as problematizações da saúde}

As relações de poder a partir da modernidade podem ser caracterizadas, segundo Foucault (2008), pela grande importância estratégica atribuída aos aspectos biológicos das pessoas enquanto seres vivos e enquanto espécie. Ele denominou esse processo de biopolítica, entendida como 
a maneira como se procurou, desde o século XVIII, racionalizar os problemas postos à prática governamental pelos fenômenos próprios de um conjunto de viventes constituídos em população: saúde, higiene, natalidade, longevidade, raças (Foucault, 2008, p. 431).

O desenvolvimento da biopolítica é indistinguível do surgimento das questões da governamentalidade do Estado moderno, com seu território e sua população, e da articulação dos saberes/poderes das ciências biomédicas, das ciências humanas e da estatística como instrumentos de racionalização dessas práticas governamentais insurgentes.

Assim, se no poder soberano medieval os tratados de política se apresentavam como conselhos ao 'príncipe', nos primórdios da modernidade esses conselhos são substituídos pelos princípios da arte de governar com a definição das razões do Estado, ou seja, pela constituição de quais são os problemas do governo e de como esses problemas devem ser geridos. Uma das principais finalidades do governo passa a ser, dessa maneira, aumentar o poder e a riqueza da nação. Rosen (1958 apud Osborne, 2005, p. 177) argumenta que, para tanto, foi necessária uma população extensa e abastecida com recursos materiais para a sua sobrevivência e expansão. Além disso, era preciso que a população estivesse sob o controle do governo, a fim de ser manipulada e utilizada segundo as necessidades das políticas públicas.

Com as artes de governar surge a noção de população, enquanto conjunto de seres viventes em um determinado território (Foucault, 2002b). É sobre essa população que o governo incide e é ela que faz parte das riquezas do Estado. Entretanto, uma população fraca e doente não tem muita capacidade de fornecer riquezas, de maneira que a saúde da população começa a ser problematizada como questão de governo.

A partir da preocupação política com a "tarefa da gestão da vida em nome do bem-estar da população como ordem vital e de cada um de seus sujeitos vivos"3 (Rose, 2001, p. 1, minha tradução), desenvolve-se a governamentalidade, conceito cunhado por Foucault para se referir ao momento de intensificação da racionalização dos governos dos Estados modernos no qual a biopolítica começa a assumir suas características. Na governamentalidade, a questão da vida é posta como assunto principal, uma vez que, se no poder soberano a morte era o momento de maior exercício de poder, na modernidade a morte passa a ser justamente o momento de fuga de qualquer poder. Dessa maneira, o governo assume como função política investir sobre a vida, para fazer viver o máximo possível.

Essas relações de poder que foram se constituindo na modernidade configuraram-se como uma espécie de poder pastoral laico: elas atravessam as pessoas tanto como indivíduos quanto como componentes de uma massa, ou seja, são relações de poder ao mesmo tempo individualizantes e massifi- 
cantes. As estratégias biopolíticas modernas, como princípios securitários que garantem a manutenção e a expansão da vida da população, surgem a partir dessa dupla perspectiva: a vida das pessoas enquanto indivíduos e a vida das pessoas enquanto membros de uma massa.

Essas insurgentes preocupações e práticas biopolíticas articularam-se com o regime disciplinar, entendido como a organização do aparato governamental em instituições que teriam por finalidade organizar as pessoas nos espaços (escola, fábrica, hospital, prisão), a fim de facilitar a vigilância e o controle (Foucault, 2002b). De acordo com Lupton, as principais estratégias disciplinares são "a observação, o exame, a medida e a comparação de indivíduos a partir de uma norma estabelecida"4 (Lupton, 2005, p. 99, minha tradução). A disciplina opera pela introdução de normas na vida social que, quando desviadas, constituem aberrações morais ou patologias que devem ser controladas, como argumenta Rose, a partir de Bauman e Agamben: "gerir a saúde do 'corpo político' requer inevitavelmente o controle e a eliminação dos 'corpos forasteiros'"5 (Bauman e Agamben, 2001, p. 2, minha tradução).

Pode-se afirmar que a concepção de saúde desenvolvida a partir dessas nascentes estratégias biopolíticas e disciplinares apresenta três características, principalmente: a saúde como dever do Estado e direito do cidadão; a saúde compreendida como ausência de doenças; e a saúde medida em termos da quantidade de anos vividos. Dessa forma, pode-se perceber uma avaliação eminentemente quantitativa, objetiva e massificante das questões relativas à saúde da população.

Até o começo do século XX, não havia maiores preocupações acerca da qualidade da vida individual das pessoas. A saúde individual era avaliada principalmente em termos da quantidade de anos vividos, e essa quantidade era medida e estendida por meio de um controle da incidência de doenças. $\mathrm{O}$ tema da qualidade era tratado apenas no que diz respeito à população como um todo, como se pode observar nos projetos biopolíticos do início do século XX que alcançaram a sua máxima manifestação no governo nazista da Alemanha (Rose, 2001). A população nacional, nesse contexto, era compreendida como a manifestação da superioridade de uma nação em competição hipotética com outras, de maneira que ela deveria ser saudável, no sentido de estar bem alimentada, bem exercitada e com a incidência de doenças bem controlada.

O controle da qualidade da população ocorreu tanto por meio do investimento do Estado em políticas de saúde e em medidas eugênicas quanto por meio do desenvolvimento e da incitação a práticas tais como o fitness. Desse modo, a biopolítica da primeira metade do século XX introduz uma novidade em relação ao século anterior: se no século XIX a saúde era uma questão eminentemente de governo, uma vez que era dever do Estado, no século XX a preocupação (assim como a responsabilidade) pela saúde começa 
a ser levemente deslocada para os indivíduos (seja pela propaganda, seja pela coerção).

\section{As estratégias biopolíticas contemporâneas}

O projeto biopolítico moderno começou a sofrer modificações a partir da segunda metade do século XX, o que pode ser relacionado a diversos movimentos, tais como os problemas surgidos nos serviços de saúde em consequência da impossibilidade de ela ser fornecida pelo Estado como uma propriedade absoluta (Osborne, 2005), e às limitações teóricas e clínicas que a conceituação exclusivamente quantitativa e objetiva da saúde estava impondo às ciências biomédicas e às políticas de saúde (Lalonde, 1981; Rose, 2007b).

Osborne (2005) argumenta que, apesar de os serviços de saúde terem assumido grande relevância como estratégia governamental, essa problematização da saúde enquanto questão de governo tornou explícito que era "simplesmente impossível instituir um determinado 'direito' à saúde"6 (Osborne, 2005, p. 179). Isso porque, por mais que fossem desenvolvidas estratégias racionais de intervenção médica no controle das doenças, ainda persistem diversos problemas de saúde que escapam dos limites do controle político e do próprio autocontrole dos indivíduos (Lalonde, 1981).

Encontra-se, então, uma limitação conceitual no projeto de manutenção da saúde da população, em decorrência dos próprios aspectos dos processos de saúde/doença (Osborne, 2005). Isso por conta de que a saúde não pode ser definida como algo que pode ser conquistado, distribuído ou controlado. Canguilhem (apud Caponi, 1997) argumenta que a noção de saúde não é algo absoluto ou determinado, que pode ser fornecido, mas que é, antes, de todo, um processo aberto de normatização. A saúde é muito mais do que oposição à doença, é capacidade de instituir normas no ambiente, processo que envolve diversas relações entre a pessoa e o ambiente. No mesmo sentido, afirma Osborne: "saúde não é um conceito absoluto ou determinado, mas um essencialmente indeterminado, relativo ou elástico"7 (2005, p. 179, minha tradução).

O projeto biopolítico moderno, ao tornar a saúde uma meta dos indivíduos e da nação, tendeu a conceber a saúde como algo absoluto. De acordo com Osborne (2005), isso fez que a saúde se deslocasse de um direito para um dever do cidadão, transformando políticas de saúde em polícias da saúde: "tais procedimentos totalizantes, em vez de instituir a saúde, aparentam apenas corromper a própria noção de saúde"8 (Osborne, 2005, p. 181, minha tradução).

As políticas de saúde no fim da primeira metade do século XX passaram, então, a evitar a absolutização da saúde como objetivo e meta. A conse- 
quência disso, argumenta Osborne (2005), foi uma modificação dos sistemas de saúde, no sentido de instituir políticas indiretas. Isso significa que o governo "não pode garantir a saúde como tal"9 (Osborne, 2005, p. 179), mas apenas fornecer as condições básicas para que a população mantenha sua saúde, dentre as quais pode-se citar a construção de hospitais, a manutenção dos sistemas de saneamento, a oferta de serviços de saúde etc.

É possível também detectar uma mudança na concepção de saúde por parte das ciências biomédicas. A saúde antes era definida como a ausência ou a aniquilação de doenças, e uma vida saudável era aquela que não padecia de doenças e que, justamente por isso, conseguia ser vivida por mais tempo (Ribeiro, 2003). A saúde era, então, problematizada de uma forma quantitativa (saúde $=$ quantidade de anos vividos). Assim, era concebida como um problema objetivo: é preciso definir o grau de incidência das doenças de uma população específica para introduzir medidas que reduzam essa incidência, a fim de que essa população viva por mais tempo possível.

Essa objetividade de análise quantitativa começou a ser questionada, como pode ser visto no enunciado de Fleck e colaboradores citados por Seidl e Zannon, porque "na busca de acrescentar anos à vida, era deixada de lado a necessidade de acrescentar vida aos anos" (2004, p. 581). De maneira que, a partir da segunda metade do século XX, inicia-se um deslocamento de uma análise quantitativa da saúde para uma análise qualitativa; a partir disso, a saúde passou a ser compreendida também enquanto bem-estar, o que foi decisivo para o desenvolvimento da noção de qualidade de vida. 10

Um documento interessante para os propósitos deste artigo e que se insere nesse duplo movimento de transformação de concepções sobre políticas de saúde e de surgimento de uma abordagem qualitativa da saúde é o relatório, produzido em 1974, por Marc Lalonde, A New Perspective on the Health of Canadians. Nesse documento, Lalonde aponta para as limitações contidas na visão tradicional de uma compreensão de política de saúde pensada/concebida unicamente como desenvolvimento de recursos, aparatos e técnicas para os serviços de saúde:

O sistema de serviços de saúde, entretanto, é apenas uma das diversas maneiras de manter e de melhorar a saúde (...). Ao mesmo tempo em que foram realizados aprimoramentos no cuidado à saúde, nas condições gerais de vida, na proteção da saúde pública e na ciência médica, forças opostas ameaçadoras atuaram para desfazer o progresso no aumento da saúde dos canadenses. Essas forças opostas constituem o lado negro do progresso econômico. Elas incluem a poluição do ambiente, a vida nas cidades, os hábitos de indolência, o abuso de álcool, tabaco e drogas, e os padrões alimentares (...). Para essas ameaças ambientais e comportamentais à saúde, o sistema organizado de serviços de saúde pode fazer pouco mais do que servir como uma rede de captação das vítimas ${ }^{11}$ (Lalonde, 1981, p. 5, minha tradução). 
Diante disso, tanto é insuficiente que as políticas de saúde se concentrem unicamente no desenvolvimento dos serviços de saúde quanto resulta impossível o governo assumir sozinho a responsabilidade pela saúde da população. Isso porque os processos de saúde/doença são concebidos como complexos, visto que envolvem diversos fatores ambientais e comportamentais que escapam ao controle direto do campo político. Assim, Lalonde (1981) procura desenvolver um novo conceito de campo da saúde que consiga, por um lado, contemplar sua complexidade de variáveis e, por outro, fragmentar a responsabilidade pela busca e manutenção dessa saúde.

O conceito de campo de saúde apresentado por Lalonde (1981) é organizado em quatro categorias: biologia humana, como as características orgânicas que influem na saúde; ambiente, como todas as variáveis que interferem na saúde que são externas ao corpo humano e sobre as quais os indivíduos têm pouco ou nenhum controle; estilos de vida, como a esfera das decisões individuais que afetam a saúde, sobre as quais há certo controle individual; e organização dos serviços de saúde, como o conjunto de recursos, técnicas e profissionais que garantem o funcionamento desses serviços.

O conceito de saúde compreendido como campo e organizado nessas categorias permite um modelo de análise mais abrangente, com base no qual é possível rastrear algumas das principais mudanças contemporâneas ocorridas na problematização da saúde. Diversas particularidades chamam a atenção no conceito de Lalonde (1981) e tornam seu texto muito útil para compreender algumas das estratégias biopolíticas atuais, dentre elas as de que: a saúde não é mais responsabilidade estrita do Estado; a preocupação pela saúde não está mais circunscrita à clínica ou ao hospital, mas antes se dissemina na vida cotidiana dos indivíduos; a introdução da categoria 'estilo de vida' traz a questão da autonomia individual, na qual se constitui todo um campo discursivo relativo à responsabilidade e à gestão de si; e a saúde não é mais analisada em termos estritamente quantitativos, mas também em seus aspectos qualitativos.

Quanto à responsabilidade do Estado pela saúde, a argumentação de Lalonde (1981) se aproxima muito daquela de Osborne (2005), no sentido de que é impossível o Estado garantir a saúde da população, sendo o papel que lhe cabe o de fornecer as mínimas garantias gerais de saúde. É possível encontrar uma análise semelhante em Rose:

Não se espera mais que o Estado resolva as demandas da sociedade por saúde. (...) Por um lado, o Estado retém a responsabilidade que adquiriu nos séculos XVIII e XIX (...) de garantir as condições gerais de saúde (...). Por outro lado, em um ambiente de promoção de saúde, o Estado tenta se libertar de algumas das responsabilidades, que ele adquiriu no século $\mathrm{XX}$, de proteger os indivíduos das consequências da doença e do acidentel2 (Rose, 2001, p. 6, minha tradução). 
A ideia organizadora dessa fragmentação da responsabilidade estatal é a de que, como diversos fatores que interferem na saúde da população são decorrentes do ambiente ou do próprio estilo de vida dos indivíduos, eles também devem responsabilizar-se pela sua saúde. Como é impossível para os médicos ou para o governo observarem os comportamentos cotidianos de todos, cada um é convidado a colaborar e instituir práticas de autocontrole. Lalonde (1981) afirma que os problemas de saúde "não podem ser solucionados unicamente pela prestação de serviços de saúde",13 mas devem ser trabalhados junto com os cidadãos, de maneira que "eles vão se tornar parceiros dos profissionais de saúde na preservação e na melhora de sua vitalidade"14 (p. 6; grifos meus, minha tradução). Rose, por sua vez, argumenta que "todo cidadão deve agora se tornar agora um parceiro ativo na busca pela saúde, aceitando suas responsabilidades em manter seu próprio bem-estar"15 (Rose, 2001, p. 6, minha tradução).

Essa ideia de parceria entre a população e os profissionais da saúde pode ser relacionada às transformações do modelo disciplinar para um modelo de controle (Deleuze, 1992). Se os processos biopolíticos modernos se desenvolveram articulados aos regimes disciplinares, em um modelo institucional (o hospital como local de cuidado de saúde) de vigilância (o médico como o responsável pela preservação da saúde) dos corpos individuais e coletivos (par indivíduo/massa), as estratégias biopolíticas contemporâneas se aproximam muito mais dos regimes de controle. Neles, as instituições não mais circunscrevem os limites da vigilância, uma vez que todos os indivíduos são tornados agentes de controle de si e do próximo. Dessa forma, não cabe mais a rígida delimitação entre os profissionais da saúde que se responsabilizam pelo atendimento e a população que recebe esse atendimento, uma vez que ambos devem trabalhar em conjunto na promoção da saúde e na prevenção dos possíveis riscos que possam acometê-la. Essa é “a nova medicina 'sem médico nem doente', que resgata doentes potenciais e sujeitos a risco" (Deleuze, 1992, p. 225).

Rabinow (1999, p. 135) afirma que, nesse processo, os polos corpo e população são rearticulados. Assim, também não faz mais sentido a distinção nítida entre os elementos da saúde de uma pessoa enquanto indivíduo e enquanto membro de uma população, uma vez que os estilos de vida de poucos indivíduos podem afetar a saúde de muitos e todos são responsáveis pela própria saúde e pela saúde dos outros. A noção de risco adquire uma importância fundamental nesse processo de rearticulação, pois o risco não incide em um indivíduo ou em uma população, mas é uma estimativa probabilística de um possível dano que pode ocorrer a um grupo de indivíduos - esses, por sua vez, fragmentados pelo próprio cálculo da presença de riscos (Castiel, 1999): 
A prevenção moderna é antes de tudo o mapeamento de riscos. O risco não é o resultado de perigos específicos colocados pela presença imediata de uma pessoa ou um grupo de pessoas, mas sim a fusão de 'fatores' impessoais que tornam um risco provável. Assim, a prevenção é a vigilância, não do indivíduo, mas sim de prováveis ocorrências de doenças, anomalias, comportamentos desviantes a serem minimizados, e de comportamentos saudáveis a serem maximizados (Rabinow, 1999, p. 145).

Rabinow afirma que o surgimento da noção de risco enfatiza "um gerenciamento administrativo preventivo de populações de risco", assim como promove "o trabalho de cada um sobre si próprio de maneira contínua" (Rabinow, 1999, p. 144). A ideia de que os indivíduos são responsáveis pela autoadministração dos riscos à sua saúde pressupõe que eles sejam autônomos. Não é a toa que Lalonde, ao comentar a categoria 'estilo de vida' em seu relatório, apele para a "fé no poder do livre arbítrio"16 (Lalonde, 1981, p. 36, minha tradução). A noção de risco se apresenta intrinsecamente articulada com a concepção iluminista da pessoa enquanto indivíduo que tem liberdade de escolha e capacidade de cálculo e projeção de seu futuro e de suas atitudes: "risco, aqui, denota um conjunto de modos de pensar e de agir, envolvendo cálculos sobre futuros prováveis no presente, acompanhados por intervenções sobre o presente, a fim de controlar esse futuro potencial"17 (Rose, 2001, p. 7, minha tradução). Assim, a rígida oposição entre saúde e doença fica comprometida a partir da emergência da noção de risco, pois ela estabelece a ideia da propensão à doença: “a distinção entre populações saudáveis e não saudáveis se dissolve totalmente, uma vez que tudo é uma fonte de 'risco', e todos podem ser vistos como 'em risco'"18 (Petersen, 2005, p. 195, minha tradução).

A aproximação da noção de risco com a concepção de autonomia individual também ressignifica a noção de saúde no sentido de possibilitar que esse indivíduo possa ser ativo na autoavaliação de seu estado de saúde. Dessa forma, passa a ser possível a introdução de variáveis subjetivas na análise da saúde, que se articula no desenvolvimento da questão do bem-estar, provocando um movimento crucial: o deslocamento de um modelo quantitativo de análise da saúde para um modelo qualitativo. Lalonde faz afirmações explícitas em relação a esse assunto, tais como "boa saúde física e mental é necessária para a qualidade de vida à qual todos aspiram"19 (Lalonde, 1981, p. 5, grifos meus, minha tradução) e "seu objetivo [do sistema de saúde] continuará a ser não apenas adicionar anos a nossas vidas, mas vida a nossos anos, de maneira que todos possam aproveitar as oportunidades oferecidas pelo progresso econômico e pelos avanços da justiça social"20 (Lalonde, 1981, p. 6, grifos meus, minha tradução).

Pode-se observar que a utilização da expressão qualidade de vida já aparece de maneira insurgente com as principais características pelas quais 
foi posteriormente definida, a saber, qualidade de vida como conjunção dos estilos de vida com as condições de vida. Qualidade de vida aparece, dessa maneira, como uma ideia básica que denota a existência de um bem-estar individual, em decorrência da possibilidade de o indivíduo conseguir fruir as condições de vida que o desenvolvimento econômico e cultural da sociedade possibilita.

Percebe-se como o tema da qualidade da população é paulatinamente substituído pelo tema da qualidade de vida individual. Esse último já surge articulado às ideias discutidas anteriormente (autonomia individual, autoadministração de riscos, responsabilidade pela saúde própria e dos outros), de maneira que o mesmo aponta para uma demanda econômica e moral. Demanda econômica porque aponta para o controle dos gastos públicos com serviços de saúde desnecessários, dado que incidem em problemas que poderiam ter sido evitados, uma vez que foram consequência de um estilo de vida 'indolente' ou 'descomprometido'. Demanda moral porque aponta para a necessidade de modificação dos estilos de vida prejudiciais à saúde, que passam a ser valorados negativamente.

\section{Usos e significados da qualidade de vida}

Minayo, Hartz e Buss (2000) argumentam que a ideia de qualidade de vida contém uma noção básica: o aproveitamento das condições culturais e econômicas que determinada sociedade oferece para os seus indivíduos. Entretanto, tal aproveitamento pode ser considerado de várias maneiras, uma vez que existem determinantes históricas (em cada momento histórico mudam as concepções do que seja uma vida boa), culturais (cada cultura valoriza de modo diferente as práticas de vida) e sociais (cada camada da sociedade valoriza práticas distintas). A noção de qualidade de vida configura-se, então, como uma relação entre a qualidade das condições de vida disponíveis (fatores ambientais, sociais e econômicos) e a qualidade dos estilos de vida escolhidos (fatores comportamentais), de maneira que é a percepção individual desses elementos que os articula.

Desse modo, é introduzida uma dimensão subjetiva na problematização da saúde, por meio da qual o indivíduo e suas percepções sobre seu estado de saúde e bem-estar são colocados em posição de destaque e de importância estratégica. Tal problematização da saúde no nível individual se manifesta de duas formas: há sempre uma possibilidade de que a saúde individual seja aprimorada, uma vez que o indivíduo tem tanto a capacidade de modificar seus estilos de vida para aumentar sua qualidade de vida quanto a capacidade de avaliar a eficácia ou não dos serviços de cuidado à saúde e as políticas de saúde. Há, também, uma consequente responsabilidade de 
perceber e dizer a verdade sobre o seu estado de saúde ou de mudar o seu estilo de vida. Decorrentes disso surgem questões acerca de por que alguns indivíduos não modificam estilos de vida que são danosos à sua saúde, ou por que alguns indivíduos não usufruem de produtos disponíveis no mercado que possibilitam uma 'fruição mais completa' da vida:

Saúde é algo que está dentro do controle do indivíduo. (...) as formas contemporâneas de bem-estar exigem cada vez mais que os indivíduos assumam uma responsabilidade pessoal por seus próprios futuros e adquiram produtos e serviços que são elaborados para atender às suas necessidades individuais. (...) as principais características das abordagens e preocupações contemporâneas nos discursos correntes sobre saúde (...) são: o indivíduo (ou self) ativo; a importância do conhecimento especializado e a crença inabalável de que nossa qualidade de vida pode ser 'melhor'21 (Nettleton, 2005, p. 208-209, grifos meus, minha tradução).

Tal expansão do campo da saúde apresenta um duplo movimento: por um lado, o poder de controle dos indivíduos sobre sua própria saúde aumenta, uma vez que ela passa a ser entendida também em termos de bem-estar e de escolhas individuais; por outro lado, cresce concomitantemente a possibilidade de esses mesmos indivíduos serem controlados, uma vez que prolifera o número de vigilantes da saúde, assim como a quantidade de dados fornecidos aos profissionais da saúde.

O processo de desenvolvimento do conceito de qualidade de vida nos últimos trinta anos mostra, de uma maneira muito interessante, esse duplo movimento. Seidl e Zannon (2004) afirmam que a qualidade de vida surge como uma definição global e abstrata de satisfação, como se pode observar na conceituação elaborada pela Organização Mundial da Saúde: “a percepção do indivíduo de sua posição na vida, no contexto da cultura e do sistema de valores em que vive e em relação aos seus objetivos, expectativas, padrões e preocupações" (apud Minayo, Hartz e Buss, 2000, p. 13).

Com base nessa noção geral, pesquisadores da área das ciências biomédicas perceberam um grande potencial em utilizar as percepções individuais como medidas confiáveis de avaliação da eficácia dos procedimentos médicos. Inicia-se, dessa forma, um processo de tentativa de refinamento dessa noção para uma definição mais conceitual e instrumental, relacionada à eficácia do tratamento e/ou das pesquisas na área da saúde. Na década de 1980 ocorre, ao lado da compreensão da qualidade de vida como noção abstrata, a tentativa de constituição de um conceito específico.

Da década de 1990 adiante, Seidl e Zannon (2004) apontam para o desenvolvimento de um consenso mínimo atingido entre os pesquisadores sobre os dois aspectos mais importantes do conceito qualidade de vida: a subjetividade e a multidimensionalidade. Em relação à subjetividade, 
“trata-se de considerar a percepção da pessoa sobre o seu estado de saúde e sobre os aspectos não médicos do seu contexto de vida" (Seidl e Zannon, 2004, p. 582). A multidimensionalidade implica que o conceito de qualidade de vida é atravessado por diversas dimensões (física, mental, ambiental, social etc.).

Da busca por uma conceituação mais precisa da qualidade de vida e por uma delimitação mais segura das dimensões que a atravessam foi elaborado o conceito de Qualidade de Vida Relacionada à Saúde (QVRS). Nele, a ênfase recai "sobre sintomas, incapacidades ou limitações ocasionados por enfermidade" (Seidl e Zannon, 2004, p. 584). O conceito de qualidade de vida relacionada à saúde, diferentemente da concepção geral, assume uma característica muito mais instrumental, uma vez que opera para fornecer dados aos profissionais da saúde sobre suas práticas.

Observa-se então, em relação ao duplo movimento de controle descrito acima, que a noção geral de qualidade de vida se relaciona, grosso modo, à capacidade de o indivíduo controlar sua própria saúde (uma vez que ela decorre, em parte, de percepções e escolhas suas). O conceito específico de qualidade de vida relacionada à saúde, por sua vez, remete mais à possibilidade de os indivíduos serem controlados em decorrência dos mesmos fatores, uma vez que suas escolhas são observadas e suas percepções são estudadas por meio de instrumentos de medida da qualidade de vida.

O uso discursivo da expressão qualidade de vida, entretanto, não se resume a essas duas possibilidades. Pode-se perceber, nos últimos trinta anos, um processo pelo qual esse termo foi semanticamente inflacionado. A qualidade de vida começou a ser impregnada dos mais diversos sentidos a ponto de ter extravasado o campo semântico da área da saúde para ser capturada pelos discursos de mercado, do marketing, da política global e das preocupações cotidianas individuais -, de maneira que se tornou cada vez mais indeterminada. A ideia de qualidade de vida passou a ser tratada de forma tão difusa que o seu próprio significado conceitual se diluiu nesta multiplicidade.

Não pretendo resgatar o sentido original da preocupação pela qualidade de vida, muito menos organizar a multiplicidade de sentidos contidos nesse termo. Porém acredito ser interessante uma tentativa de apresentação da variedade de usos contemporâneos da expressão qualidade de vida, dentre os quais é possível localizar: conceito: uso técnico referente à percepção individual do estado de saúde dentro das condições econômicas e sociais existentes (qualidade de vida = estilos de vida + condições de vida); parâmetro: a partir do que a expressão assume características mais político-administrativas, no sentido de operar como medida de avaliação dos níveis de saúde de determinada população e das políticas de saúde; dado: significado atribuído pelos resultados dos instrumentos de medida de qualidade de vida; instrumento/método: uso para a elaboração dos testes de eficácia de 
intervenções ou de procedimentos médicos; e um aspecto da realidade: um sentido oposto aos anteriores, visto que aqui, em vez de meio, a qualidade de vida é apresentada como uma meta ou um fim que devem ser alcançados.

Diante desses diversos usos contemporâneos da expressão qualidade de vida, concentro-me, agora, na sua perspectiva como meta a ser alcançada, a fim de realizar algumas considerações sobre o tema da responsabilização individual pela própria saúde. No próximo tópico me deterei na qualidade de vida enquanto parâmetro, enquanto dado e enquanto instrumento para analisar a questão da medida da qualidade de vida.

\section{Responsabilidade individual pela própria saúde}

O tema da responsabilidade individual pela própria saúde articula-se com distintos campos (político-administrativo, econômico, moral), assim como se relaciona intimamente com uma compreensão cultural de pessoa enquanto indivíduo autônomo. Sua ideia básica parte da constatação de que existem diversos fatores que afetam a saúde e que vão para além do controle político (uma vez que dizem respeito a fatores ambientais e comportamentais). Dada a fragmentação das variáveis relativas aos processos de saúde/doença, é possível ocorrer uma fragmentação análoga na responsabilidade pela manutenção dessa saúde. Uma vez que as escolhas que os indivíduos livremente realizam interferem na sua saúde, eles devem ser responsabilizados por suas escolhas. Assim, a responsabilidade pela promoção de saúde "está largamente dispersa entre cidadãos individuais, governos, profissionais da saúde e instituições"22 (Lalonde, 1981, p. 34, grifos meus, minha tradução).

A responsabilidade pela saúde assume um aspecto tanto econômico quanto moral. Economicamente, trata-se de uma otimização, por parte do Estado, da utilização de recursos na área de saúde. Assim, uma vez que algumas doenças são causadas também pelos comportamentos individuais, o custo do tratamento deve ser arcado por esses indivíduos. Foi exatamente essa lógica que propiciou o desenvolvimento e a generalização dos sistemas privados de saúde, instrumentalizados pelo cálculo de predisposição a riscos.

A passagem da lógica econômica para a lógica moral é tão sutil que nem se faz sentir na maioria das vezes: uma vez que os estilos de vida que oferecem risco à saúde individual acarretam custos, esses estilos passam a ser valorados negativamente como ruins (são danosos, custosos e, além disso, errados). Nessa correlação, compõe-se um imperativo moral pela saúde: se a medicina está avançada a ponto de indicar estimativas confiáveis acerca dos riscos que cada prática acarreta para a saúde, se esses danos que possivelmente podem ocorrer à saúde podem ser evitados por práticas de autocuidado e autocontrole, por que ainda existem indivíduos que insistem em se expor voluntariamente a riscos? 
A exposição voluntária a riscos e a prática de estilos de vida 'não saudáveis' começam a ser entendidas como erros morais ou falhas próprias. A moralização dessas condutas opera de maneira a elaborar uma estratégia de controle dos comportamentos individuais que passa pela própria consciência dos indivíduos, ou seja, pelo autocontrole. Pouco a pouco disseminam-se certos discursos sobre saúde que, ao se tornarem hegemônicos, transformam a qualidade de vida em uma meta a ser atingida. O interessante a notar é que ocorre, dessa forma, um deslocamento da questão da qualidade de vida enquanto percepção subjetiva para dever individual. A busca pela qualidade assume características de purificação moral da vida, ao passo que, quando alguém não consegue viver uma vida com qualidade, ele é o único culpado por isso.

Ao transformar a qualidade de vida em uma meta a ser alcançada, sob pena de punições morais, a própria noção de saúde transforma-se em algo absoluto. Como já citei anteriormente, a absolutização da saúde transforma-a de direito em dever do cidadão (Osborne, 2005). Essa imposição da qualidade de vida contribui para o desenvolvimento de uma moral da boa forma, como afirma Sibilia:

Todo um grupo de valores extremamente atuais se alinha em torno da nova moral da boa forma, que exige não apenas autoestima, bem estar e qualidade de vida, mas também originalidade, êxito e alto desempenho em todos os âmbitos (...) esse empreendimento requer uma extenuante gestão de si mesmo, que inclui tanto o indispensável autocontrole como a cotidiana adesão aos estilos de vida considerados $\operatorname{corretos}^{23}$ (Sibilia, 2010, p. 2-3, minha tradução).

Diante do imperativo de ser saudável e da imposição da qualidade de vida, surge a culpa. Spink (2009) observa que esse é o lado 'interno' da responsabilidade, e mostra como a responsabilidade pela própria saúde incute uma lógica de dever nas pessoas com base na qual qualquer prejuízo passa a ser compreendido como falta, erro ou descomprometimento, posto que "esses estilos de vida saudável tornaram-se um dever para consigo mesmo, em relação aos nossos entes queridos e para com o coletivo" (Spink, 2009, p. 24).

Um dos exemplos mais radicais dessa articulação entre a responsabilidade econômica e o dever moral pela própria saúde pode ser encontrado no artigo "Convergência de planos de previdência e planos de saúde: uma realidade cada vez mais próxima", no qual Hummel argumenta que "mais cedo do que se imagina os usuários de planos de saúde que também tenham um plano de previdência privada estarão assinando um único contrato, e vice-versa" (2006, p. 25). Isso ocorrerá, argumenta o autor, em decorrência da necessidade de se compreender a saúde de forma preventiva e individual, para que possam ser elaboradas estratégias que responsabilizem os indivíduos pela sua própria saúde, desenvolvendo neles práticas de autocontrole: 
(...) a única forma de resolver a avalanche deficitária dos modelos de saúde é a ampla conscientização de que cada cidadão está 'condenado' a cuidar e zelar pela sua própria saúde. (...) cuidar da saúde não é dever do Estado, ou de qualquer outro membro da cadeia de assistência, mas do próprio indivíduo. O caos crescente nos sistemas de saúde só será equacionado quando a 'doença' encontrar um obstáculo: o 'doente' (Hummel, 2006, p. 25; grifos meus).

Pode-se observar como o discurso sobre a responsabilidade individual pela própria saúde, e pela saúde dos outros, adquire, para além do caráter econômico, um caráter moral, principalmente ao se analisar tal responsabilidade pelo seu inverso, a irresponsabilidade. Irresponsabilidade passa a significar tanto o fato de não ter sido responsável com a própria saúde, no caráter estratégico de não ter realizado os devidos cálculos perante os riscos presentes, quanto no caráter moral por não ter sido prudente o suficiente perante os próprios atos. O irresponsável o é tanto em relação à sua própria vida quanto em relação à sociedade como um todo. O irresponsável é, diante de tantos meios de informação disponíveis, um ignorante, mas não um ignorante no sentido do não saber, mas no sentido de saber e, mesmo assim, deliberadamente não agir. Assim, é moralmente ignorante:

O dramático é saber que $75 \%$ dessas doenças [as cardiovasculares] ocorrem por novos hábitos da sociedade e pela ignorância (ou displicência) do indivíduo com relação a sua saúde. (...) Criamos uma sociedade pateticamente irresponsável com a sua própria saúde. (...) tirando a legião de excluídos que habita o planeta e que não têm comida, educação e as mínimas condições higiênicas e sanitárias, a grande maioria do restante da população é 'irresponsável' com a sua saúde e a daqueles que dele dependem (notadamente, sua família) (Hummel, 2006, p. 25-26; grifos meus).

Hummel (2006) aponta, então, para três formas de sanar esse círculo vicioso de ignorância e displicência, por meio do desenvolvimento de práticas de prevenção: educacional: mediante campanhas publicitárias, da conscientização de comunidades e da introdução de matérias sobre o tema da saúde nas escolas; compulsória: por meio da criação de mecanismos legais de controle da saúde, como proibição de certos comportamentos danosos à saúde, a partir do que o Estado ditaria 'as regras de conduta'; e mercadológica, mediante a oferta de benefícios monetários para as pessoas que adotarem práticas de prevenção.

$\mathrm{Na}$ argumentação de Hummel, aparecem alguns dos principais elementos nos quais o debate sobre a responsabilização individual pela própria saúde é posto em movimento. Em termos da moralização cada vez mais crescente do tema da saúde e do cuidado próprio, cria-se um terreno fértil para o crescimento de uma noção de saúde não mais enquanto direito, mas como 
dever do cidadão, um dever para consigo mesmo e para com os outros. Uma vez que esse dever é atravessado pela liberdade de escolha dos indivíduos de se adequarem ou não a essa norma, surge concomitantemente a questão de como lidar com os 'desviantes'.

Outra consequência dessas lógicas é a transformação da saúde em um bem, pois, como afirma Hummel (2006), a "doença não vale a pena". A noção de prevenção é ressignificada em termos econômicos, a partir do que se compreende que o ato de prevenir as doenças "pode lhe render benefícios financeiros ao final da vida" (Hummel, 2006, p. 27). A saúde, assim, passa a assumir características de uma bioeconomia (Spink, 2007).

Então temos que, paralela a essa motivação racional e lógica do custo e benefício, opera-se uma purificação moral, mascara-se uma afronta à liberdade e empreende-se uma ousada cruzada contra a doença. O não saudável passa a ser, em consequência, o negligente, o irresponsável, o ignorante ou o administrador incompetente.

\section{A medida da qualidade de vida}

Um dos aspectos mais importantes nos diversos usos discursivos da expressão qualidade de vida é a existência de diversos instrumentos que se propõem a medi-la. Seidl e Zannon (2004) comentam uma pesquisa na qual foram identificados "446 instrumentos, no período de setenta anos. No entanto, 322 instrumentos identificados, equivalentes a mais de 70,0\% do total, apareceram na literatura a partir dos anos $80^{\prime \prime}$ (2004, p. 581).

Como argumentei no tópico anterior, os instrumentos de medida da qualidade de vida se relacionam com três dos usos sugeridos dessa expressão: como um parâmetro, como um dado e como um método. A medida da qualidade de vida enquanto parâmetro permite a análise de políticas de saúde (caráter político-administrativo); a qualidade de vida como dado oferece uma medida da saúde percebida (caráter subjetivo); por sua vez, a qualidade de vida enquanto método instrumentaliza a análise da eficácia das intervenções e procedimentos médicos (caráter técnico-científico). Entretanto, essa divisão em três usos é apenas analítica, uma vez que os instrumentos de medida da qualidade de vida apresentam esse conceito na sua forma multidimensional.

O deslocamento qualitativo ocorrido nas ciências biomédicas tal como apresentado acima influenciou fortemente não só os novos modelos de análise de saúde, como também as políticas de saúde. A partir dele o tema da qualidade de vida dos indivíduos de um país começa a adquirir relevância em nível político-administrativo. Minayo, Hartz e Buss (2000) e Matta (2005) afirmam que a própria criação do Índice de Desenvolvimento Humano 
(IDH) pode ser localizada nesse movimento, uma vez que ele pode ser caracterizado como uma tentativa de introduzir uma perspectiva qualitativa na análise, a fim de ser um índice mais completo do que a simples medida do Produto Interno Bruto (PIB).

No campo mais específico da saúde, uma vez que a qualidade de vida se tornou um aspecto fundamental na sua avaliação, os profissionais e os cientistas dessa área começaram a instrumentalizar esse conceito de maneira a utilizá-lo para fins de aprimoramento das práticas biomédicas e das políticas de saúde. O desenvolvimento de instrumentos para medir a qualidade de vida, assim como outros instrumentos psicométricos, colocou em movimento uma questão crucial: como é possível medir objetivamente percepções individuais? Dois caminhos possíveis de análise surgem, dependendo da maneira como são compreendidas as noções de objetividade e de subjetividade. Tradicionalmente, a oposição entre objetividade e subjetividade remete a duas concepções básicas. Por um lado, o objetivo pode ser compreendido como aquilo que pode ser controlado, em oposição ao subjetivo, que escapa do controle; por outro, o objetivo pode ser compreendido como aquilo que é público (no sentido de que todos têm acesso a) e em oposição ao subjetivo, que é privado (no sentido de que é íntimo). Acredito que ambas as oposições estão sendo esvaziadas em favor de uma rearticulação desses sentidos.

Como procurei argumentar quando abordei a passagem dos regimes disciplinares para os de controle (Deleuze, 1992), o controle não mais remete à ideia de um agente de controle incidindo sobre um sujeito controlado, uma vez que tal noção se disseminou nas relações sociais, de modo que todos nos transformamos em agentes de controle de nós mesmos e do outro. Diante disso, não faz mais sentido opor a objetividade à subjetividade em termos de capacidade ou não de controle, uma vez que, nos dias atuais, as próprias subjetividades se constituem com base num princípio de controle próprio.

Adicionalmente, a subjetividade não é mais compreendida como interioridade. A noção de intimidade ou de privacidade, ou seja, do subjetivo como aquilo que é oculto, também está perdendo sentido, dado que os modos de subjetivação contemporâneos têm sido cada vez mais atravessados por um processo de exposição, de espetacularização e de publicização (Ortega, 2008). Como exemplo simples desse processo, pode-se citar a substituição dos diários (enquanto arquivos secretos das intimidades pessoais) pelos blogs, orkut e facebook (arquivos públicos das vidas individuais, abertos para livre acesso).

Essa discussão sobre objetividade/subjetividade posta em movimento em relação ao desenvolvimento dos instrumentos de medida de qualidade de vida também se insere no movimento mais amplo de rearticulação dos usos e significados do que seja subjetivo ou objetivo: ao responder um teste, um paciente expõe (torna público) suas percepções subjetivas, ao mesmo 
tempo em que os 'dados objetivos' desses testes são elaborados com base nessas percepções subjetivas.

Apesar de esses instrumentos focalizarem a percepção individual do estado de saúde, seu comprometimento não é com o indivíduo. Os indicadores de qualidade de vida, argumenta Matta (2005), operam um duplo corte, em relação à população e em relação ao indivíduo:

(...) o objetivo desses estudos [em relação à população] não é avaliar a saúde da população propriamente dita, mas a performance dos sistemas de saúde através da saúde da população, ou melhor, a qualidade de vida relacionada à saúde. Os problemas tratados nesses estudos são de distribuição de recursos, financiamento, acesso aos serviços de saúde, o custo social das enfermidades e a comparação das performances dos sistemas nacionais de saúde (Matta, 2005, p. 120).

(...) [em relação ao indivíduo] o foco não é o sistema de saúde, nem a população, e podemos dizer, nem o indivíduo, mas sim a relação entre doença, tecnologia médica e seus resultados (Matta, 2005, p. 120).

Dessa maneira, os instrumentos de medida da qualidade de vida se apropriam da subjetividade dos indivíduos, não por estarem comprometidos com essa subjetividade, mas, antes, por poderem instrumentalizá-la a favor de uma racionalização maior das técnicas de promoção de saúde.

Outro aspecto interessante relacionado à medida da qualidade de vida é a sua consonância com os movimentos hegemônicos de globalização. A Organização Mundial de Saúde (OMS) instituiu, na década de 1990, uma comissão específica (o WHOQOL Group) para estudos relativos ao tema da qualidade de vida. A partir dela foi elaborado um instrumento de medida de qualidade de vida com pretensões globais: o World Health Organization Quality of Life (WHOQOL). Em uma das publicações do Whoqol Group é possível ler que "a iniciativa da OMS em desenvolver uma avaliação da qualidade de vida surge da necessidade por uma medida genuinamente internacional da qualidade de vida e por um comprometimento com a contínua promoção de uma abordagem holística da saúde e do cuidado à saúde"24 (WHOQOL Group, 1996, p. 5, minha tradução).

Essa avaliação tanto na sua forma completa, WHOQOL-100, quanto na sua forma reduzida, WHOQOL-Bref, foi elaborada em diferentes contextos nacionais e passou a ser utilizada como instrumento global de medida de qualidade de vida em contextos distintos, com os mais variados fins:

as avaliações do WHOQOL serão utilizadas de formas bem abrangentes. Elas serão de uso considerável na prática clínica (...). Para a pesquisa epidemiológica, as avaliações do WHOQOL vão permitir um acúmulo de dados detalhados de qualidade 
de vida para populações específicas, facilitando o entendimento das doenças e o desenvolvimento de métodos de tratamento. Os estudos epidemiológicos internacionais que serão tornados possíveis por instrumentos como o WHOQOL-100 e o WHOQOL-Bref vão tornar possível a realização de uma pesquisa multinacional sobre qualidade de vida, assim como a comparação dos resultados obtidos em distintos locais. (...) É antecipado que no futuro o WHOQOL-100 e o WHOQOL-Bref serão úteis na pesquisa sobre políticas de saúde e comporão um aspecto importante na rotina dos serviços de saúde e de assistência25 (WHOQOL-Group, 1996, p. 9-10, minha tradução).

A pretensão contida no projeto, como argumenta Matta (2005), é a da criação de diretrizes mundiais de uma saúde global, sendo que a qualidade de vida opera nesse movimento como parâmetro de análise e de comparação:

Entendemos que o WHOQOL é um dos instrumentos produzidos pela OMS [Organização Mundial da Saúde] para a construção de base de evidências e que constitui uma das estratégias retóricas fundamentais para a formulação de políticas de saúde internacionais e para a formação de consensos no cenário sanitário nacional e global (Matta, 2005, p. 66).

Percebe-se como a qualidade de vida tem sido uma noção muito útil para legitimar práticas, instituir medidas, organizar parâmetros, delimitar políticas, incitar consumos etc., de maneira que é, como concebe Matta, uma "estratégia de construção de evidências para o mundo globalizado" (2005, p. 102).

\section{Considerações finais}

Probleminhas terrenos: quem vive mais, morre menos? Millôr Fernandes

Procurei apresentar uma discussão da qualidade de vida que abrangesse, pelo menos em parte, a complexidade de temas que se relacionam a ela na sua dispersão discursiva. Em decorrência disso, as análises aqui desenvolvidas buscam situar os diversos usos e significados da expressão qualidade de vida, demonstrando a variedade de temas e a densidade de níveis nos quais a qualidade de vida é apresentada, visto que se articula numa rede de correlação de forças. Não procurei traçar um quadro detalhado de toda essa complexidade, uma vez que isso demandaria um esforço ainda maior, mas, 
antes, indicar os possíveis caminhos pelos quais um estudo mais aprofundado pode seguir.

É importante observar como o tema da qualidade de vida é composto no século XX em relação a diversas problemáticas, sendo rapidamente inflacionado com os mais diversos significados e instrumentalizado das mais diversas maneiras. A qualidade de vida é um termo que aglutina facilmente muitos significados, processo esse que indica o quanto a expressão está em sintonia com as demandas econômicas, políticas, culturais e científicas dos nossos dias. Dessa forma, analisar os processos por meio dos quais a qualidade de vida foi povoada de significados e instrumentalizada em índices, medidas, dados, conceitos etc. é uma maneira de compreender a complexidade das relações sociais contemporâneas, uma vez que nenhuma expressão adquire sentido à toa, mas somente na medida em que atende a demandas histórica e culturalmente situadas.

Outro elemento importante a ser ressaltado é a maneira como o uso cotidiano hegemônico da expressão qualidade de vida vem operando na direção de uma totalização da saúde. De fato, pode-se observar como a saúde se transformou em um 'valor incontroverso' (Azize, 2002). Tem se tornado cada vez mais difícil encontrar indivíduos que discordem de ideias tais como a de que a saúde é o nosso principal bem, ou de que devemos investir em nossa saúde, ou de que devemos fazer de tudo para conquistar uma boa saúde. O que interessa nesse fato não é a preocupação com a saúde em si, mas antes a maneira em que essa preocupação é veiculada e os usos que dela se faz. Como procurei discutir, a saúde, ao ser problematizada como um valor em si mesmo, é totalizada como uma meta a ser alcançada. Ocorre, assim, uma inversão: a saúde já não é um meio pelo qual podemos levar uma vida boa, mas antes é a própria finalidade de nossas vidas. A saúde, ao ser transformada em meta absoluta, em 'valor incontroverso', desloca-se facilmente para um princípio totalitário e moralizador. Constitui-se não só uma moral da boa forma por meio da qual ocorre uma imposição da felicidade e do bem-estar, mas também todo um novo nicho de mercado, repleto de produtos que se utilizam da classificação 'saudável' para seduzir os consumidores.

A mídia hegemônica utiliza largamente a imposição da saúde como bem inquestionável. É o que se pode observar pela depreciação que ocorre com os 'não saudáveis': fumar, ser melancólico, ser obeso, não praticar intensamente exercícios físicos está fora de moda nos dias de hoje. Essa ditadura da saúde acaba por se relacionar com uma percepção de que nossa saúde pode ser sempre melhor, que sempre podemos aprimorar nossa qualidade de vida por meio da compra de alguns produtos ou de modificações e adequações nos nossos estilos de vida. A saúde, como valor absoluto, transforma-se em saúde ideal ou saúde perfeita, a partir do que os indivíduos são levados a permanecer constantemente insatisfeitos com o seu atual estado de saúde, e 
isso favorece o desenvolvimento de uma 'indústria da insatisfação' (Sibilia, 2010). Paradoxalmente, os discursos hegemônicos da qualidade de vida acabam operando como os discursos da falta de qualidade de nossas vidas atuais.

Ao situar os discursos e práticas hegemônicos de qualidade de vida na complexa rede de problematizações contemporâneas, procurei desnaturalizá-la enquanto assunto universal. Isso pode ser compreendido como uma tentativa de resistir à concepção hegemônica de saúde e de vida com qualidade, a partir da qual outros modos de ser, assim como outros modos de ser saudável, possam, também, ser possiveis.

\section{Nota do editor}

O presente trabalho foi resultado de uma pesquisa de iniciação científica realizada sob a orientação da professora Martha Ramirez-Gálvez, e fez parte do projeto “Corpos e Tecno/Máquinas: Risco, Consumo e Marcadores de Diferenças", vinculado ao departamento de Ciências Sociais da Universidade Estadual de Londrina.

\section{Notas}

I Graduado em Psicologia pela Universidade Estadual de Londrina (UEL), Londrina, Paraná, Brasil. Mestrando em Psicologia Social e Institucional pela Universidade Federal do Rio Grande do Sul (UFRGS). <gfgimenes@gmail.com>

Correspondência: Universidade Federal do Rio Grande do Sul, Instituto de Psicologia, Departamento de Psicologia Social e Institucional, Rua Ramiro Barcelos, 2.600, Santa Cecília, CEP 90350-003, Porto Alegre, Brasil.

2 No original: "We have become the kinds of people who think of our present and our future in terms of the quality of our individual biological lives."

3 No original: "the task of the management of life in the name of the well-being of the population as a vital order and of each of its living subjects."

4 No original: "observation, examination, measurement and the comparison of individuals against an established norm." 
5 No original: "to manage the health of the 'body politic' inescably requires the control and elimination of 'foreign bodies'."

${ }^{6}$ No original: “just impossible to impose a certain 'right' do health."

7 No original: "health is not an absolute or determinate concept but an essentially indeterminate, relative or elastic one."

8 No original: "Such totalising procedures, instead of instituting health, seem only to corrupt the very notion of health."

9 No original: "cannot guarantee health as such."

10 Insere-se, nesse movimento, a criação da Organização Mundial da Saúde em 1948, a partir da qual foi elaborada uma definição 'oficial' da saúde nos seguintes termos: "estado de completo bem-estar físico, mental e social, e não somente a ausência de enfermidade ou invalidez".

11 No original: "The health care system, however, is only one of many ways of maintaining and improving health. (...) At the same time as improvements have been made in health care, in the general standard of living, in public health protection and in medical science, ominous counter-forces have been at work to undo progress in raising the health status of Canadians. These counter-forces constitute the dark side of economic progress. They include environmental pollution, city living, habits of indolence, the abuse of alcohol, tobacco and drugs, and eating patterns (...). For these environmental and behavioural threats to health, the organized health care system can do little more than serve as a catchment net for the victims."

12 No original: “The state is no longer expected to resolve society's needs for health. (...) On the one hand, the state retains the responsibility that it acquired in the 18th or 19th century (...) to secure the general conditions for health (...). On the other hand, within such a health-promoting habitat, the state tries to free itself of some of the responsibilities that it acquired in the 20th century for securing individuals against the consequences of illness and accident."

13 No original: "cannot be solved solely by providing health services."

14 No original: "they will themselves become partners with health professionals in the preservation and enhancement of their vitality."

15 No original: "Every citizen must now become an active partner in the drive for health, accepting their responsibility for securing their own well-being."

16 No original: “(...) in favour of faith in the power of free will."

17 No original: “Risk here denotes a family of ways of thinking and acting, involving calculations about probable futures in the present followed by intervention into the present in order to control that potential future."

18 No original: "the distinction between health and unhealthy populations totally dissolves since everything is a source of 'risk' and everyone can be seen to be 'at risk'." 
19 No original: “(...) good physical and mental health are necessary for the quality of life to which everyone aspires."

20 No original: "Its goal will continue to be not only to add years to our life but life to our years, so that all can enjoy the opportunities offered by increased economic and social justice."

${ }^{21}$ No original: "Health is something which lies within the control of the individual (...) contemporary forms of welfare are increasingly requiring that individuals take personal responsibility for their own future and purchase goods and services which are designed to meet their personal requirements (...) the key characteristics of contemporary approaches and preoccupations within current discourses on health (...) are: the active individual or 'the self'; the salience of 'expertise'; and the unshakeable belief that our quality of life can be 'better'."

22 No original: “(..) is widely dispersed among individual citizens, governments, health professions and institutions."

23 No original: "Todo un cortejo de valores sumamente actuales se alinea en torno a la flamante moral de la buena forma, que exige no sólo autoestima, bienestar y calidad de vida, sino también originalidad, éxito y alto desempeño en todos los ámbitos. (...) ese emprendimiento requiere una extenuante gestión de sí mismo, que incluye tanto el indispensable autocontrol como la cotidiana adhesión a los estilos de vida considerados correctos."

24 No original: "WHO's initiative to develop a quality of life assessment arises from a need for a genuinely international measure of quality of life and a commitment to the continued promotion of an holistic approach to health and health care."

25 No original: "the WHOQOL assessments will be used in broad-ranging ways. They will be of considerable use in clinical trials (...) For epidemiological research, the WHOQOL assessments will allow detailed quality of life data to be gathered on a particular population, facilitating the understanding of diseases, and the development of treatment methods. The international epidemiological studies that would be enabled by instruments such as the WHOQOL-100 and the WHOQOL-BREF will make it possible to carry out multi-centre quality of life research, and to compare results obtained in different centres (...) It is anticipated that in the future the WHOQOL-100 and the WHOQOL-BREF will prove useful in health policy research and will make up an important aspect of the routine auditing of health and social services." 


\section{Referências}

AZIZE, Rogério L. A química da qualidade de vida: um olhar antropológico sobre o uso de medicamentos e saúde em classes médias urbanas brasileiras. 2002. Dissertação (Mestrado Antropologia Social) - UFSC, Florianópolis, 2002.

BELASCO, Angélica G. S.; SESSO, Ricardo C. C. Qualidade de vida: princípios, focos de estudo e intervenções. In: DINIZ, Denise P.; SCHOR, Nestor. (Org.). Guia de qualidade de vida. Barueri: Manole, 2006.

BLAY, Sergio L.; MERLIN, Marina S. Desenho e metodologia de pesquisa em qualidade de vida. In: DINIZ, Denise P.; SCHOR, Nestor. (Org.). Guia de qualidade de vida. Barueri: Manole, 2006.

CASTIEL, Luis D. A medida do possível: saúde, risco e tecnobiociências. Rio de Janeiro: Editora Fiocruz, 1999.

CAPONI, Sandra. Georges Canguilhem y el estatuto epistemológico del concepto de salud. História, Ciências, Saúde: Manguinhos, Rio de Janeiro, v. 4, n. 2, p. 287-307, jul./out. 1997.

DELEUZE, Gilles. Post-scriptum sobre as sociedades de controle. In: Conversações. São Paulo: Editora 34, 1992.

FOUCAULT, Michel. Em defesa da sociedade. São Paulo: Martins Fontes, 2002a.

História da sexualidade. Rio de Janeiro: Graal, 2003. (a vontade de saber; v. 1).

- Microfísica do poder. Rio de Janeiro: Graal, 1982.

. Segurança, território, população.

São Paulo: Martins Fontes, 2002b.

Nascimento da biopolítica. São Paulo: Martins Fontes, 2008.

GASTALDO, Denise. Is Health Education Good for You? Re-thinking Health Educa- tion through the Concept of Bio-power. In: PETERSEN, Alan; BUNTON, Robin. (Org.). Foucault, Health and Medicine. Nova York: Routledge, 2005. P. 113-133.

HUMMEL, Guilherme S. Convergência de planos de previdência e planos de saúde: uma realidade cada vez mais próxima. Apólice: revista do mercado de seguros, São Paulo, v. 11, n. 101, p. 25-27, 2006.

LALONDE, Marc. A New Perspective on the Health of Canadians: a Working Document. Ottawa: Government of Canada, 1981.

LUPTON, Dora. Foucault and the Medicalisation Critique. In: PETERSEN, Alan; BUNTON, Robin. (Org.). Foucault, Health and Medicine. Nova York: Routledge, 2005. P. 94-111.

MATTA, Gustavo C. A medida política da vida: a invenção do WHOQOL e a construção de políticas de saúde globais. 2005. Tese (Doutorado em Saúde Coletiva) - Instituto de Medicina Social, Universidade do Estado do Rio de Janeiro, Rio de Janeiro, 2005.

MINAYO, Maria C. S.; HARTZ, Zulmira M. A.; BUSS, Paulo M. Qualidade de vida e saúde: um debate necessário. Ciência \& Saúde Coletiva, Rio de Janeiro, v. 5, n. 1, p. 7-18, 2000.

NETTLETON, Sarah. Governing the Risky Self: How to Become Healthy, Wealthy and Wise. In: PETERSEN, Alan; BUNTON, Robin. (Org.). Foucault, Health and Medicine. Nova York: Routledge, 2005. P. 207-222.

ORTEGA, Francisco. O corpo incerto: corporeidade, tecnologias médicas e cultura contemporânea. Rio de Janeiro: Garamond, 2008.

OSBORNE, Thomas. Of Health and Statecraft. In: PETERSEN, Alan; BUNTON, R. (Org.). Foucault, Health and Medicine. Nova York: Routledge, 2005, p. 173-188.

PETERSEN, Alan. Risk, governance and the new public health. In: ; BUNTON 
Robin. (Org.). Foucault, Health and Medicine. Nova York: Routledge, 2005. p. 189-206.

RABINOW, Paul. Artificialidade e iluminismo: da sociobiologia à biossociabilidade. In: Antropologia da razão. Rio de Janeiro: Relume Dumará, 1999. p. 135-158.

RIBEIRO, Renato J. Novas fronteiras entre natureza e cultura. In: NOVAES, Adauto (Org.). O homem-máquina: a ciência manipula o corpo. São Paulo: Companhia das Letras, 2003.

ROSE, Nikolas. The Politics of Life Itself. Theory, Culture \& Society, Nottingham, v. 18, n. 6, p. 1-30, 2001.

Beyond Medicalisation. The Lancet, Londres, n. 369, p. 700-702, 24 Feb. 2007a.

The Politics of Life Itself: Biomedicine, Power and Subjectivity in the Twenty-First Century. Princeton: Princeton University Press, 2007b.

SEATTLE QUALITY OF LIFE GROUP. About the WHOQOL Instruments. Seattle: Seattle Quality of Life Group, [s.d.]. Disponível em: $<$ http://depts.washington.edu/yqol/docs/ WHOQOL_Info.pdf>. Acesso em: 17 jan. 2013.

SEIDL, Eliane; ZANNON, Célia. Qualidade de vida e saúde: aspectos conceituais e metodológicos. Cadernos de Saúde Pública, Rio de Janeiro, v. 20, n. 2, p. 580-588, mar./abr. 2004.
SIBILIA, Paula. ¿Cómo ser un cuerpo contemporáneo? Autoestima, bienestar, creatividad, alto desempeño y buena forma. Cuadernos del Inadi, Buenos Aires, n. 2, p. 1-7, ago. 2010. Disponível em: <http://cuadernos.inadi. gob.ar/numero-02/paula-sibilia-como-serun-cuerpo-contemporaneo $>$. Acesso em: 18 out. 2010.

SPINK, Mary J. A saúde na encruzilhada entre biopolítica e bioeconomia: reflexões sobre os paradoxos da "era dos direitos" na globalização hegemônica. In: RIBEIRO, Maria A. T. R.; BERNARDES, Jefferson de S.; LANG, Charles E. (Org.). A produção na diversidade: compromissos éticos e políticos em Psicologia. São Paulo: Casa do Psicólogo, 2007. p. 55-74.

Estilos de vida saudável e práticas de existência: fronteiras e conflitos. In: BERNARDES, Jefferson; MEDRADO, Benedito. (Org.). Psicologia social e politicas de existência: fronteiras e conflitos. Maceió: Associação Brasileira de Psicologia Social, 2009. p. 15-26.

WORLD HEALTH ORGANIZATION. WHOQOL-Bref: Introduction, Administration, Scoring and Generic Version of the Assessment. Genebra: World Health Organization, dez.1996. Disponível em: $<$ www.who.int/mental_health/media/en/ 76.pdf>. Acesso em: 17 jan. 2013.

Recebido em 18/05/2011

Aprovado em 26/11/2012 